\title{
Gridifying the TINKER Conformer Generator Application for gLite Grid
}

\author{
Attila Kertész ${ }^{1}$, Ferenc Ötvös ${ }^{2}$, and Péter Kacsuk ${ }^{1}$ \\ 1 MTA SZTAKI Computer and Automation Research Institute \\ H-1518 Budapest, P.O. Box 63, Hungary \\ \{attila.kertesz, kacsuk\}@sztaki.hu \\ 2 Biological Research Center \\ H-6701 Szeged, P.O. Box 521, Hungary \\ otvos@brc.hu
}

\begin{abstract}
Grid Computing provides an efficient way for parallelizing and gridifying computationally and data intensive applications of various research fields. One of these application areas is molecular dynamics. In this paper we examine a biochemical application that generates conformers by unconstrained molecular dynamics at high temperature to overcome conformational bias then finishes each conformer by simulated annealing and energy minimization to obtain reliable structures. We provide a general way for turning biochemical applications into Grid workflows that can be executed by Grid portals exploiting the computational power of available production Grids. First we describe the gridification process, then provide experimental results that show the achieved speedup of the ported application.
\end{abstract}

Keywords: Grid Computing, Grid portal, molecule conformer generation, TINKER application.

\section{Introduction}

E-Science infrastructures play an important role in enabling large-scale scientific research. In order to establish such e-infrastructures, various Grid systems have been created and run in production as a service for the scientific community. While several years ago users and companies having computation and data intensive applications looked sceptical at the forerunners of Grid solutions, Grid Computing 3] has become a separate research field: currently Grids are targeted by many world-wide projects. Research groups were forming around specific middleware components and different research branches have arisen. Many user groups from various research fields put their trust in Grids, and usage statistics and research results show that they were undoubtedly right. Nowadays research directions are focusing on user needs, therefore more efficient utilization and interoperability play the key roles. The current wave of Grid research targets user support 4. Though several production Grid solutions are available today (eg. 1], 9], 8]), the application of these systems is still not widespread. 
Nevertheless we already have good examples that show how beneficial these systems can be [11, especially for biology, chemistry and physics.

In this paper we examine a biochemical application that generates conformers of flexible molecules, here a tetrapeptide (Tyr-Pro-Phe-Phe-NH2), by unconstrained molecular dynamics at high temperature to overcome conformational bias then finishes each conformer by different statistical modeling (SM) methods to obtain reliable structures. These structures were successfully used to obtain the active conformation of the peptide for its receptor ( $\mathrm{mu}$ opioid receptor) by QSAR modeling assisted with an efficient variable selection algorithm. We provide a way for turning biochemical applications into Grid workflows that can be managed and executed through high level graphical interfaces offered by Grid portals exploiting the computational power of available production Grids. Regarding related works, Valverde in [13] has already shown, how to execute TINKER binaries [12] in EGEE Grids [1, but this solution used only low-level, command line interface. On the contrary, we propose a general, high-level solution using Grid portals, which provide graphical user interface for non-Grid expert users. The general gridification process we introduce in this paper can be applied to any parallelizable application, but the final Grid workflow is highly dependent on the structure (the number and order of algorithms used) of the appropriate application.

The rest of the paper is organized as follows. In Section 2 we introduce the biochemical application and discuss its requirements. In Section 3 we introduce our approach for gridification, and in Section 4 we present the evaluation result of our proposed solution. Finally, we conclude our paper in Section 5 .

\section{The TINKER Conformer Generator Application}

The application (shown in Figure 1) generates conformers by unconstrained molecular dynamics at high temperature to overcome conformational bias $(\mathrm{T})$ then finishes each conformer by simulated annealing and/or energy minimization to obtain reliable structures. The parameter files contain reference for the molecular force field (here Amber99), vacuum/implicit water (here GBSA) environment, target temperatures, etc. The aim is to obtain conformation ensembles to be evaluated by multivariate statistical modeling. It uses the TINKER library 12. for molecular modeling for further QSAR studies and drug development. The target end users are biologists or chemists, who need to examine molecule conformers with the TINKER package. The conformer generation algorithm in its present form comprises five different conformer finishing methods:

(i) minimizing the initial conformational states generated at high temperature (TM),

(ii) performing a short low temperature (e.g. $300 \mathrm{~K}$ ) dynamics with the high temperature conformations to simulate a low temperature thermodynamical ensemble (TD),

(iii) minimizing the above low temperature states (TDM), 


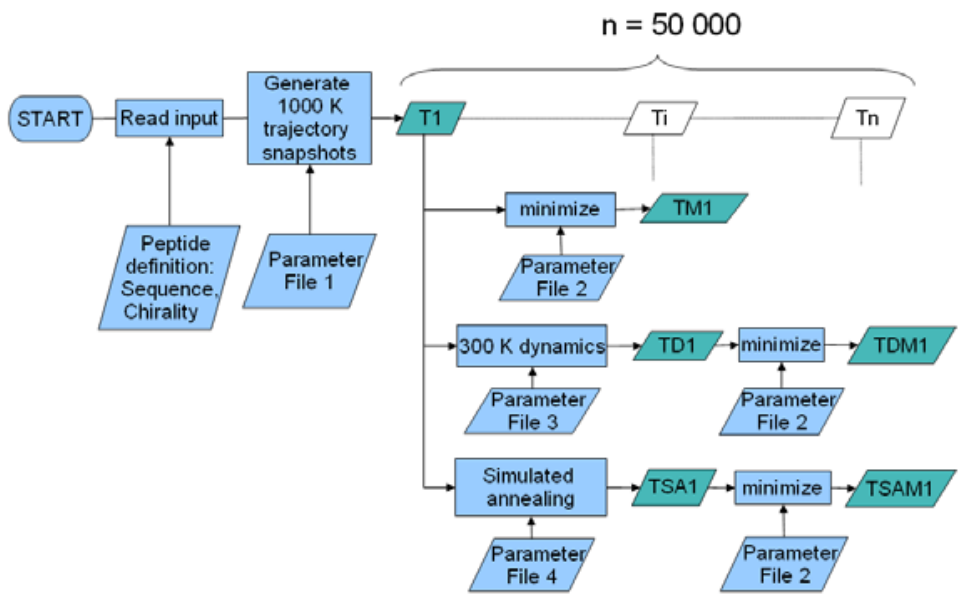

Fig. 1. TINKER Conformer Generator application

(iv) cooling the high temperature states by simulated annealing, e.g. to $50 \mathrm{~K}$, or completely to $0 \mathrm{~K}$ (TSA),

(v) minimizing the annealed states (TSAM).

The reason why to generate the conformational states or conformers (which are conformational states at some local energy minima) is to investigate which of them suits better for the subsequent multivariate statistical modeling (namely quantitative structure-activity relationships studies, QSAR), then the algorithm may be simplified. Our most recent successful QSAR modeling makes use of the TSAM structures which is the most computationally costly method, but may serve as a reference method to obtain the most reliable thermodynamical ensembles. Regarding execution times, these are the average run times (in hours) of the various methods in the vacuum environment used in the application on a single $2 \mathrm{GHz}$ CPU machine: $\mathrm{T}-13, \mathrm{TM}-28$, TD -3 , TDM -28 , TSA - 26 and TSAM -28 (the abbreviations discussed in this paragraph correspond to the ones shown in Figure 1). If we use the implicit water (GBSA) environment, the execution of the different steps takes 1,5 times longer. This means that the execution of the whole application takes around 5-8 days.

\section{Gridifying the Application}

The P-GRADE Grid Portal [6] is a workflow-oriented grid portal with the main goal to support all stages of grid workflow development and execution processes. It enables the graphical design of workflows created from various types of executable components (sequential and parallel jobs), executing these workflows in Globus-based computational Grids relying on user credentials, and finally, analyzing the monitored trace-data by the built-in visualization facilities. 
The P-GRADE Portal provides the following functions: defining grid environments, creation and modification of workflow applications, managing grid certificates, controlling the execution of workflow applications on grid resources and monitoring and visualizing the progress of workflows and their component jobs.

A P-GRADE Portal workflow is a directed acyclic graph (DAG) that connects sequential and parallel programs into an interoperating set of jobs. The nodes of such a graph are batch jobs, while the arc connections define data relations among these jobs. Arcs define the execution order of the jobs and the input/output dependencies that must be resolved by the workflow manager during execution. The semantics of the workflow execution means that a node (job) of the workflow can be executed if, and only if all of its input files are available, i.e., all the jobs that produce input files for this job have successfully terminated, and all the other input files are available on the Portal Server and at the pre-defined storage resources. Therefore, the workflow describes both the control-flow and the data-flow of the application. If all the necessary input files are available for a job, then the workflow manager transfers these files - together with the binary executable - to the computational resource where the job is allocated for execution. Managing the transfer of files and recognition of the availability of the necessary files is the task of the workflow manager component of the Portal Server.

The WS-PGRADE Portal [7] is the latest member of the P-GRADE portal family. It is a web-based frontend of the gUSE infrastructure 5, which is a lose collection of web services supporting high-level distributed deployment and scalability. It supports development and submission of distributed applications executed on the computational resources of production Grids. The workflow semantics of gUSE builds on the successful concept of the original P-GRADE Portal, but it also provides several new features: Job-wise parametrization gives a flexible and computing efficient way of parameter study (or sweep) (PS) applications, permitting the submissions of different jobs in different numbers within the same workflow. During workflow execution, so-called workflow instances are created. In this way different submissions can be managed and tracked of the same concrete workflow. Web service calls and different sub-workflows can also be embedded into the jobs/nodes of the workflow.

The original application has been gridified by creating P-GRADE and WSPGRADE workflows shown in Figure 2. Though these two versions of the application workflow are similar, we present both versions in order to exemplify gridification in both portals. The open-source P-GRADE portal can be attractive for many scientists, while the WS-PGRADE portal has some new enhancements and provide further development support. The grid workflow application consists of three phases (denoted by dashed areas in the figures):

1. The first phase is a generator job responsible for the generation of input data for parameter studies in the next phase.

2. The second phase consist of a PS sub-workflow, in which three PS jobs are defined for executing three different algorithms (discussed in Section 2), and an additional PS job that collects the outputs of the three threads and copies 
them in a compressed file to a pre-defined remote storage. (In the P-GRADE workflow we can see an additional job as the first job of the sub-workflow that is responsible for copying the PS input files one-by-one from a remote storage (created by the generator job in the first phase) to the other three PS jobs. There is no need for such a job in the WS-PGRADE portal, because this feature is supported by its workflow interpreter.)

3. Finally in the third phase, a collector job gathers the output files of the PS sub-workflows and uploads them in a single compressed file to the remote storage, which can be easily downloaded by the user.

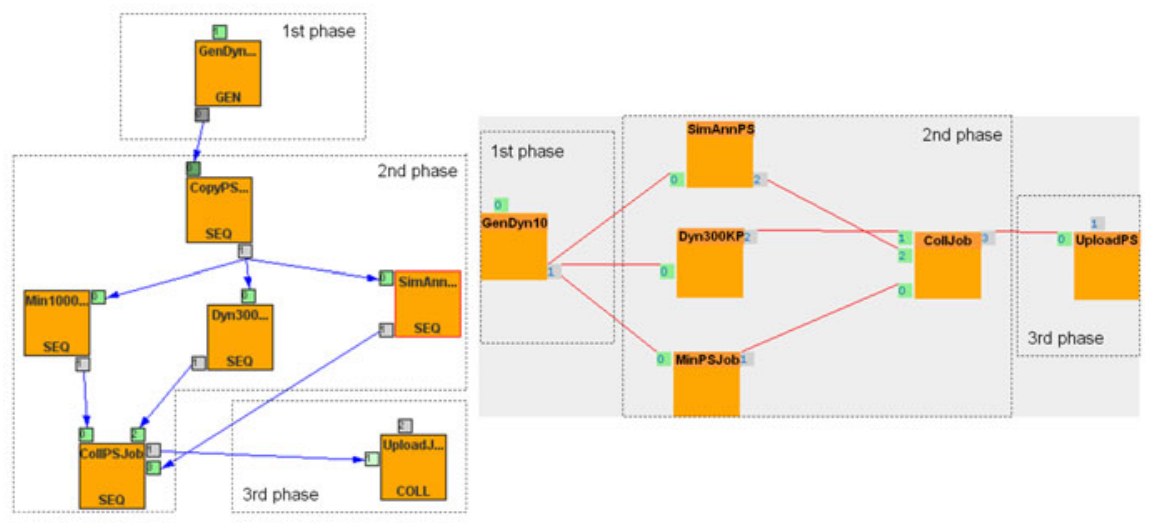

Fig. 2. TINKER Conformer Generator workflow in the P-GRADE and WS-PGRADE portals

In the original sequential application, the TINKER algorithms are executed by Bash Unix scripts, which are also applicable in EGEE infrastructures 11. The sequential generation of 50000 conformers cannot be parallelized, this is done by the first generator job. This job has an input file containing the required binaries of the TINKER library and a user script that perform the execution of the TINKER algorithms (which comes from the original user application). During execution it reads the arguments (that can be used for parameterizing the workflow) and generates the conformers, then sorts them to 50 output files containing 1000 conformers, the TINKER library and the pre-defined parameters. Finally these files are uploaded to a pre-defined remote storage. These files represent the input files of the parameter study sub-workflow instances.

The second phase of the workflow application is parallelized by the parameter study construct. Instead of executing the three TINKER algorithms (minimization, dynamics and simulated annealing) sequentially on 50000 conformers, we decided to divide the computation into several parts. Since performing an algorithm on one conformer takes less then a second and thousands of jobs may flood the Grid resources, we decided to pack 1000 conformers to feed sequentially to the algorithms, and gathered the algorithms into three threads. Each thread processes 1000 conformers 50 times, therefore this decomposition resulted in 3 
times 50 parameter study jobs. After the execution of the TINKER algorithms, the output of these threads are gathered, compressed and copied to a storage by the last job of this phase. Since these PS jobs are executed 50 times parallelly (for each input file), this phase means 200 job submissions. (In the P-GRADE portal version we have an additional copy PS job, which makes 250 total job submissions in this phase.) Finally in the third phase the last collector job of the workflow compresses all the output files into a single result file, and uploads it to a storage. Altogether the gridified workflow application consists of 202 jobs in the WS-PGRADE and 252 jobs in the P-GRADE portals.

Regarding execution times, the original application runs for about a week on a general single CPU machine. Regarding our experiences the generator job of the workflow run for 9-18 hours (depending on the speed of the actual execution environment), while the parameter study jobs executing the TINKER algorithms on 1000 conformers run within the range of 30-60 minutes each. The total execution time includes additional queuing, communication and file transfer delays. Regarding data handling: the input TINKER package is $4.5 \mathrm{MBs}$. The generator job creates 50 tarballs containing 1000 conformers each, and the TINKER package. Each one of these files are around 6.1 MBs. The output of the PS jobs varies from 1 to $2 \mathrm{MBs}$, and the final compressed result file is around $280 \mathrm{MBs}$ (which is proportional to the number of conformers (50000) and the size of the molecule (80 atoms)). One QSAR study multiplies this data, because it needs 15-20 molecules as a minimum to simulate.

Our main tasks in application porting were to find the acceptable decomposition, to modify the original application scripts to work separately with a selected algorithm on the decomposed input sets, and to create additional wrapper scripts that set up the working environment on the selected grid resources right before executing the algorithms, and clear up the working directory after execution. After the scripts have been created, we designed the workflow structure and set its properties according to the available Grid environments (further described in the next Section). In the preliminary tests we experienced that some Grid resources failed to execute the TINKER binaries correctly. Later we found out that the invoked Fortran programs could not manage files with long path names (eg. /var/scratch/jobs/540873.ce.ui.savba.sk/ https_3a_2f_2flb2.egee.ces-net.cz_3a9000_2f1wvjmGCIwWLIDrsdmH8yg), which is typical in the utilized VOs. First we used the default HOME of the resources (which are usually different from the pre-defined working directories), but in some cases we still experienced failures, because sometimes more jobs were sent to different cores of the same host and tried to use the same working directories. The final solution was to create unique temporary working directories (for which we used the mktemp Unix command).

The application is the first part of a drug design toolbox, whose first step is to generate high number of conformers of extremely flexible molecules (in our case it is a tetrapeptide). The description of highly flexible molecules can only be considered adequate when they are treated as thermodynamical ensembles and the rules of statistical thermodynamics are applied. Therefore a successful QSAR study on this kind of molecules can only be performed when they are 
treated as statistical ensembles and this kind of molecular description serves as an input for the multivariate statistical analysis step. As a result, we will use the gridified application to perform several QSAR studies (including 15-20 molecule explorations each).

\section{Performance Evaluation}

In order to execute applications (and use any service of production Grids) scientists have to acquire Grid certificates from a certificate authority (eg. 2] ) and register themselves to the selected Grids or Virtual Organizations (VO). We have applied for membership into two multidisciplinary and one biomedical application specific VOs of EGEE for evaluation and further utilization: VOCE [14, SEEGRID 10] and BIOMED [15. We have executed the workflow several times in all three VOs. The total execution time (the makespan) varied between one and three days. The actual execution time is highly dependent on the actual load of the resources in the utilized VO. The summary of the average execution times can be seen in Figure 3 on the left.

By the time we carried out these experiments, we could reach 21 resources in VOCE, 41 in SEEGRID and 183 in BIOMED. During the evaluation we experienced a little load on VOCE, medium on SEEGRID and high on BIOMED. Regarding reliability, no execution errors happened in VOCE, but we encountered several resubmissions in SEEGRID and some in BIOMED. These environmental conditions affected the measured values. As a result we can state that the gridified application can be executed 4 to 7 times faster on real production Grids compared to general single CPU machines. Finally we can see the detailed execution times of different workflow application phases for selected evaluation runs in Figure 3 on the right.
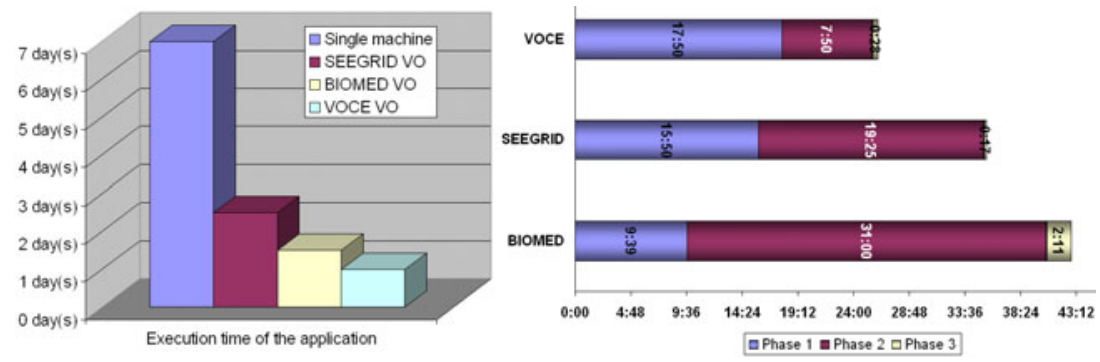

Fig. 3. Evaluation of the application on different Grid infrastructures

\section{Conclusions}

In this paper we have shown how to port a legacy biochemical application to a parameterizable Grid workflow that can be executed by non-Grid expert users 
from Grid portals providing a graphical user interface for production Grids. We have successfully parallelized the application and evaluated it on three Virtual Organizations of the EGEE Grid. The presented results show that we have achieved a significant speed-up with at most 7 times faster execution compared to general single CPU machines. Though the presented worklows have been designed for EGEE VOs, using the gUSE infrastructure it is possible to execute the workflow in different Grids (eg. GT4, BOINC). Our future work aims at creating a graphical portlet for workflow submissions in the P-GRADE and WSPGRADE portals, which will further simplify user interactions with production Grids.

\section{Acknowledgement}

The research leading to these results has received funding from EGI-InSPIRE project (contract number RI-261323) and the Enabling Grids for E-sciencE project (contract number INFSO-RI-031688).

\section{References}

1. Enabling Grids for E-sciencE (EGEE) project website, http://public.eu-egee.org/

2. e-Science grid authentication in Europe, http://www.eugridpma.org/

3. Foster, I., Kesselman, C.: The Grid 2: Blueprint for a New Computing Infrastructure. Morgan Kaufmann Publishers Inc., San Francisco (2003)

4. Grid Application Support Centre website, http://www.lpds.sztaki.hu/gasuc/

5. Grid User Support Environment (gUSE) website, http://www.guse.hu/

6. Kacsuk, P., Sipos, G.: Multi-Grid, Multi-User Workflows in the P-GRADE Grid Portal. Journal of Grid Computing, 1-18 (February 2006)

7. Kacsuk, P., Karóczkai, K., Hermann, G., Sipos, G., Kovács, J.: WS-PGRADE: Supporting parameter sweep applications in workflows. In: Proc. of the 3rd Workshop on Workflows in Support of Large-Scale Science (in conjunction with SC 2008), Austin (2008)

8. Open Science Grid (OSG) website, http://www.opensciencegrid.org/

9. National Grid Service (NGS) website, http://www.ngs.ac.uk/

10. South Eastern European Grid-Enabled eInfrastructure Development project (SEE-GRID) website, http://www.see-grid.eu/

11. Tantoso, E., et al.: Molecular Docking, an example of Grid enabled applications. New Generation Computing 22(2) (2004)

12. TINKER molecular modeling software website, http://dasher.wustl.edu/tinker/

13. Valverde, J.R.: Simplifying job management on the Grid. EMBnet.news 14(2), 25$32(2008)$

14. Virtual Organisation for Central Europe (VOCE) website, http://egee.cesnet.cz/en/voce/

15. Virtual Organisation for biomedical applications (BIOMED) website, https://twiki.cern.ch/twiki/bin/view/EGEE/LifeSciences 